\title{
Exploring the barriers of patients with diabetic foot complications in China: a qualitative interview study
}

\author{
Xiaoli Liu ${ }^{1}$, Hongling Chu ${ }^{1}$, Jinghui Zhao ${ }^{2}$, Rui Qiao ${ }^{3}$, Yuqiang Liu ${ }^{4}$, Nan Li $^{1}$, Lin Zeng ${ }^{1}$, Xiaoxiao Wang ${ }^{1}$, \\ Liyuan Tao ${ }^{1}$, Hua Zhang ${ }^{1}$, Yanyan Shi ${ }^{1}$, Lin Zhuo ${ }^{1}$, Long Zhang ${ }^{2}$, Yiming Zhao ${ }^{1}$ \\ ${ }^{1}$ Research Center of Clinical Epidemiology, Peking University Third Hospital, Beijing, China; ${ }^{2}$ Department of Interventional Radiology and \\ Vascular Surgery, Wound Healing Center, Peking University Third Hospital, Beijing, China; ${ }^{3}$ Baotou Medical College, Inner Mongolia University \\ of Science and Technology, Baotou, China; ${ }^{4}$ Department of Pharmacy, Changzhi People's Hospital, Changzhi, China \\ Contributions: (I) Conception and design: X Liu, L Zhang, Y Zhao; (II) Administrative support: None; (III) Provision of study materials or patients: \\ L Zhang, J Zhao; (IV) Collection and assembly of data: X Liu; (V) Data analysis and interpretation: X Liu, L Zhang; (VI) Manuscript writing: All \\ authors; (VII) Final approval of manuscript: All authors. \\ Correspondence to: Yiming Zhao, PhD. Research Center of Clinical Epidemiology, Peking University Third Hospital, Beijing, China. \\ Email: yimingzhao117@163.com; Long Zhang, PhD. Department of Interventional Radiology and Vascular Surgery, Wound Healing Center, Peking \\ University Third Hospital, Beijing, China. Email: longzh2000@163.com.
}

\begin{abstract}
Background: Patients with diabetic foot complications are associated with high rates of morbidity, disability, and mortality. Through findings of qualitative interviews with patients with this disease, we aimed to explore the barriers they encountered, provide evidence to improve the efficacy of medical services, discuss prevention and treatment strategies for future policymakers, and attract widespread attention from the Chinese society.

Methods: Patients with diabetic foot complications were recruited from three tertiary hospitals in China between July to September 2020. Patients were included who had a clinical diagnosis of diabetic foot complications, were 18 years or older, spoke Chinese as their first language, and were willing to share treatment experiences. An interview guide was used during the in-person semi-structured interviews that lasted 20 to 50 minutes and were audio-recorded. Transcripts were analyzed for qualitative themes.

Results: Forty-one patients (range, 38-79 years; 12 men, 29 women) were recruited. Data analysis indicated five thematic dimensions on barriers encountered by patients with diabetic foot complications: hospital visits, doctor-patient communication, mental burden, economic burden, and social support.

Conclusions: Patients with diabetic foot complications face serious financial, mental, and social burdens in China. Future studies can use the five dimensions to help solve the existing problems and improve treatment outcomes of this patient population.
\end{abstract}

Keywords: Diabetic foot complications; patients; barriers; qualitative research; China

Submitted Oct 25, 2020. Accepted for publication Feb 10, 2021.

doi: 10.21037/atm-20-7569

View this article at: http://dx.doi.org/10.21037/atm-20-7569

\section{Introduction}

Diabetic foot complications in patients lead to considerable morbidity, disability, and mortality (1). The global prevalence of diabetic foot ulcers and amputations is approximately $1.7 \%$ to $11.9 \%$ and $1.3 \%$ to $6.7 \%$, respectively (2). In China, the annual incidence of diabetic foot ulcers and amputations among patients with diabetes is $8.1 \%$ and $5.1 \%$ respectively, and the fatality rate among patients with ulcers is $14.4 \%$ (3). The recurrence rate of diabetic foot ulcers is as high as $50 \%$ after 3 years (2).

These high rates of morbidity, disability, and mortality in Chinese patients with diabetic foot complications have brought a great burden to patients, their families, 
and society. Although the global prevalence of diabetes increases annually and currently exceeds $9 \%(>11 \%$ in China), medical and social systems have not yet formed a comprehensive treatment response $(4,5)$. Identifying the specific needs of patients with diabetic foot complications could help build a professional response system of social medicine and reduce the social burden of this disease on patients.

Quantitative studies on patients with diabetic foot complications have identified the following risk factors of disability and mortality: male sex, smoking, high thrombocyte levels, diabetic nephropathy, and peripheral arterial disease $(1,3,6-8)$. In addition, studies outside China have shown that delayed visits, patient dissatisfaction with treatment outcomes, poor care of diabetic foot complications, a sense of hopelessness in diabetic patients with foot issues, and a negative patient outlook on future treatments might contribute to the high disability and mortality rates (9-14). China, a country with a large population, is in the process of reforming its healthcare system; thereby, relevant studies on Chinese patients are urgently needed.

Currently, China has a three-tier medical system; the higher the level, the stronger the ability to treat diseases (15). Although first-level hospitals are numerous and spread throughout the country, they mainly provide vaccinations, routine diagnoses, and treatment of chronic diseases and cannot diagnose and treat major diseases (e.g., severe diabetic foot complications) (15-17). The issue with diabetic foot complications is that the onset begins as a small cut but can develop within days into a fatal ulcer requiring amputation. If the patient, the patient's family, society, and the primary hospital are not identified in time, treatment could be delayed, resulting in complex and challenging follow-up treatments and an increasing risk of amputation and death (18). It is important for providers to understand the personal experience of patients with diabetic foot complications during diagnoses and treatments, identifying their common needs and difficulties, and being aware of a patient's relationship with the current medical system to help improve the prognosis and efficacy of the Chinese medical system.

Few qualitative studies have explored treatment difficulties and barriers regarding patients with diabetic foot complications. These studies only discussed diabetic foot amputations or problems in diabetic foot complications care, and thus results are relatively one-sided $(11,12,19-21)$. Currently, only one study has explored patient experiences with diabetic foot complications, but that study was performed in Canada in 2005, and the prevalence of diabetic foot complications since then has increased considerably $(14,22)$. Notably, no research in this area has been conducted in China.

Further research is warranted to understand the problems of patients with diabetic foot complications in China. The current study used qualitative methods to conduct in-depth interviews among patients with diabetic foot complications to explore the treatment barriers in China. The purpose of our study was to provide evidence in support of improving the treatment outcomes and efficacy of medical services, discuss prevention and treatment strategies of patients with diabetic foot complications for future policymakers, and attract widespread attention from Chinese society.

We present the following article in accordance with the MDAR and COREQ reporting checklist (available at http://dx.doi.org/10.21037/atm-20-7569).

\section{Methods}

\section{Study design}

This qualitative study used semi-structured interviews with open and structured questions and prompts. The interview guide was developed through literature review and expert discussion. The interviews were conducted from July to September 2020. The study was conducted in accordance with the Declaration of Helsinki (as revised in 2013). Ethical approval was obtained from Peking University Third Hospital Institutional Review Board (No. IRB00006761-M2020003). All study participants signed written informed consent forms and agreed to be recorded during the interview. The research is reported in accordance with the Consolidated Criteria for Reporting Qualitative Research (23).

\section{Study setting and recruitment}

The research was performed in three first-class tertiary hospitals in Beijing: Peking University Third Hospital, Beijing Hospital, and Beijing Shijitan Hospital. The three hospitals were selected for several reasons. First, they were independent and operate independently of each other. Second, they were large general hospitals equipped with diagnosis and treatment centers for diabetic foot complications. Each hospital treated diabetic foot complications with multidisciplinary cooperation, and 
all offered interventional vascular surgery specializing in the diagnosis and treatment of lower limb ischemia. Third, all three hospitals planned to improve their current treatment of diabetic foot complications and were willing to participate in the study.

Patients were recruited over 7 weeks from July 27 to September 13, 2020 from the three hospitals. The participating patients were identified as those who saw specialists or general doctors to treat diabetic foot complications. Patient recruitment ended after reaching thematic saturation when no new themes were identified. Purposive sampling was used in this study. The target population was patients receiving treatment for diabetic foot complications.

Patient inclusion criteria were those with a clinical diagnosis of diabetic foot complications (i.e., infection, ulceration, or destruction of foot tissues, usually accompanied by neuropathy and peripheral arterial disease) and type 1 or 2 diabetes treated conservatively or surgically, with an age of 18 years or older, having Chinese as their first language, willing to share experiences with diabetic foot complication treatments; consenting to a recorded interview; and voluntarily signing an informed consent.

Patient exclusion criteria were those with depression, mania, mental disorders, and other psychiatric diseases, and those unable to communicate without assistance, that had dementia, a speech impairment, foot problems for reasons other than diabetes, and those deemed by the clinical staff to be unable to participate in the interview.

\section{Data collection}

Patient interviews were first conducted in the Wound Treatment Center of Peking University Third Hospital. After the patient interview information was saturated, the interviews were conducted in Beijing Shijitan Hospital and Beijing Hospital. The semi-structured, face-to-face individual interviews were conducted by an experienced qualitative researcher with a $\mathrm{PhD}$ degree from a medical background (LXL). LXL, a female person, had a systematic understanding and training of the study, but was not a direct participant in the patient's treatment process. During the interview, LXL tried to be objective, without taking a personal stand or bias. The interview guide (Supplementary file 1) includes a patient's experience from symptom onset to presentation at a hospital, the difficulties and obstacles encountered by the patient during the treatment, and their opinions and suggestions.

Before the formal interview, we first established a good relationship with the patients through communication and improved our understanding of each other. At the same time, we explained the purpose and content of the study so that patients could have a detailed understanding of our study. All interviews were conducted in a quiet and private space in the hospital, allowing interviewees to share their views freely and confidentially without being influenced by others. The interview guide was used flexibly to keep the natural flow of conversation and allow patients to discuss their own experiences freely. Interviews were audiorecorded and transcribed verbatim. Each interview was 20 to 50 minutes. All the patients we investigated participated in the study, and no one refused or dropped out. We did not conduct repeated interviews with the patients.

\section{Data analysis}

All interviews were transcribed by the interviewer (LXL) verbatim. Inductive thematic analysis was adopted to generate overarching themes and subthemes. The analysis was a process of coding that does not fit data into a preexisting model or frame but rather was data-driven. This approach provided detailed descriptions of the data based on the views of patients with diabetic foot complications who had unique and personal healthcare experiences.

Nvivo V.11 software (QSR International, Melbourne, Australia) was used to help analyze data. Two researchers (LXL and ZL) independently coded and analyzed the transcripts by familiarizing themselves with the materials, generating initial codes, searching for themes, naming and defining themes, reviewing themes, and finally generating the report. To ensure reliability and effectiveness, LXL and ZL first coded the transcripts of three patients independently, and then discussed the coding and classification of the transcripts and reached an agreement. During the coding process, LXL and ZL held regular meetings to discuss and identify themes in the remaining transcripts. Interviews and reviews of transcripts continued until no new themes emerged, at which point it was determined that the themes had theoretically reached saturation. The final themes were discussed and explained by all research team members in the discussion meeting, and a consensus was reached on the main themes and relationships of the data. 
Table 1 Description of the characteristics of patients with diabetic foot

\begin{tabular}{lc}
\hline Characteristics & $\mathrm{N}(\%)(\mathrm{N}=41)$ \\
\hline Gender & $29(70.7)$ \\
Male & $12(20.3)$ \\
Female & \\
Age (years) & $1(2.4)$ \\
$30-39$ & $6(14.6)$ \\
$40-49$ & $8(19.5)$ \\
$50-59$ & $17(41.5)$ \\
$60-69$ & $9(22.0)$ \\
$70-79$ &
\end{tabular}

Education

Primary school

Junior middle school

$16(39.0)$

High school

College and above

Occupation

Retired

Paid employment

$11(26.8)$

Unemployed

$6(14.6)$

Duration of diabetes (years)

1-9

10-19

17 (41.5)

20-

Marital status

Married

Separated/divorced

Single

$1(2.4)$

Widowed

Foot status

First DFU

Recurrent DFU

$11(26.8)$

Major amputation

$1(2.4)$

Minor amputation

$14(34.1)$

Healed

8 (19.5)
Table 1 (continued)

\begin{tabular}{lc}
\hline Characteristics & $\mathrm{N}(\%)(\mathrm{N}=41)$ \\
\hline Classification of the diabetic foot & $2(4.9)$ \\
Ischaemic foot & $31(75.6)$ \\
Neuroischaemic foot & $8(19.5)$ \\
Neuropathic foot & \\
Comorbidity & $11(26.8)$ \\
Cardiovascular disease & $8(19.5)$ \\
Renal disease & $4(9.8)$ \\
Retinopathy & $19(46.3)$ \\
Hypertension & $9(22.0)$ \\
Hyperlipidemia & $4(9.8)$ \\
Cerebral infarction & \\
Medical costs & $7(17.1)$ \\
Self-paying & $34(82.9)$ \\
Medical insurance
\end{tabular}

Minor amputation refers to amputation below ankle level and major amputation refers to amputation above ankle level. DFU, diabetic foot ulcer.

\section{Results}

\section{Patient characteristics}

A total of 41 patients with diabetic foot complications in the Beijing area were recruited and interviewed between July and September 2020 (Table 1). Patients were generally middle-aged with type 2 diabetes. Thirty-one patients were interviewed at the Peking University Third Hospital, and five patients each were interviewed at the Beijing Shijitan Hospital and Beijing Hospital.

Table 2 summarizes the categories, themes, and subthemes. The supporting quotes for each theme are provided in Supplementary file 2.

\section{Patient-reported barriers}

Interview results yielded five dimensions regarding the problems and obstacles encountered by patients with diabetic foot complications: hospital visits, doctor-patient communication, mental burden, economic burden, and social support. 
Table 2 Themes and subthemes extracted from the barriers encountered by patients with diabetic foot

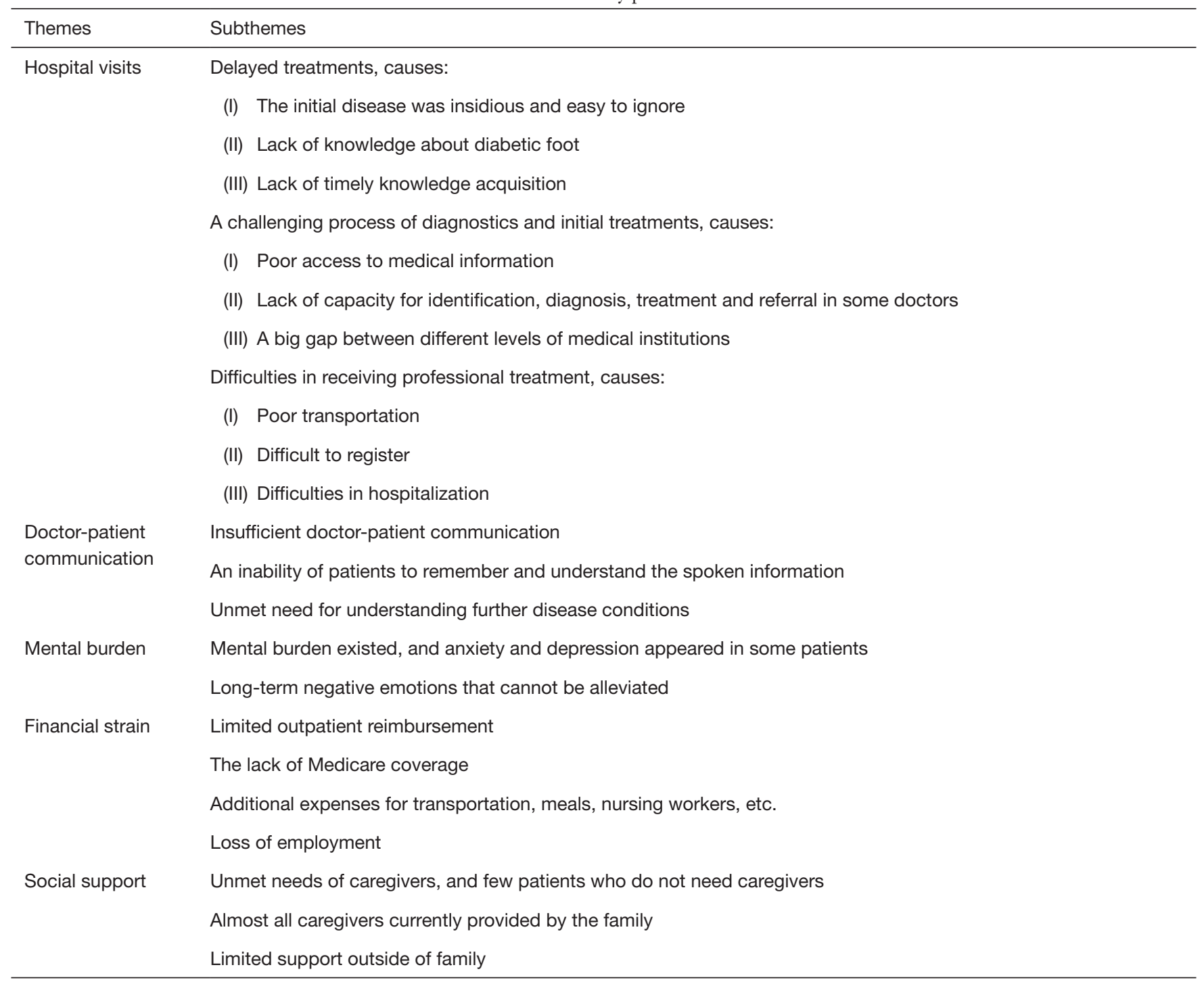

\section{Hospital visits}

Patients with diabetic foot complications encountered problems in seeking medical treatments, including delayed visits, challenging diagnostic processes and initial treatments, and difficulties in receiving professional treatments.

\section{Delayed treatments}

Some patients reported a certain degree of delay in seeing a doctor from the onset of symptoms to hospital treatment, and some would go to the hospital for treatments that last few months to half a year from onset of symptoms. Generally, they went to the hospital after the feet became re-aggravated, usually showing signs of leg swelling, odor, and blackened feet.

The primary reason for treatment delays was that the initial stage of diabetic foot complications was relatively insidious, and thus, easily ignored by patients. A female patient in her 60s reported the following:

"At the beginning, I didn't notice the swollen leg or a wound on my foot, because I could walk. Because of diabetes, my peripheral nerves are bad and I cannot see clearly. I cannot see or feel pain. When my daughter came to see me, she found that my leg was swollen and there was a small hole in the sole of my right foot... 28 days had already passed when my daughter found these problems; and then, she burriedly took me to the hospital." 
Other important reasons for treatment delays were a lack of knowledge about diabetic foot complications, having a false understanding, or not knowing the severity of diabetic foot complications, which ultimately delayed the treatment. A male patient in his 40s noted the following:

"When my right foot first started to turn black, I thought it was bruised and okay. I felt it would just get better little by little. After more than a month, my right foot did not get better, and I thought something might be wrong, so I went to the hospital to have it examined."

In addition, many patients did not acquire knowledge of diabetic foot complications in a timely manner. Most patients did not understand diabetic foot complications until symptom onset, and some patients lacked knowledge even after the symptoms began. A man in his 60s and woman in her 70s made the following comments following, respectively:

"I did not know about diabetic foot complications before. After I came here, the doctor told me that I [had] diabetic foot complications. I didn't know it before."

"I did not know what this disease was before, and now I do not know what it is. I just need to know bow to eat, and other things are not clear. My daughter knows these things. I just want to get better soon."

\section{A challenging process of diagnostics and initial treatments}

Some patients would move from one hospital to another or from one department to another before finding a professional doctor in a professional hospital. Patients presented at multiple hospitals and departments, causing an aggravation of the illness and delay in treatment. The main reason for going to multiple hospitals was that patients did not obtain correct and accurate medical information for diabetic foot complications. Most patients did not know where to go to see a doctor after symptom onset, and only after going to several hospitals did, they find a hospital that specifically treated diabetic foot complications. A woman in her 60s noted the following:

"My foot was so painful that I went to the community hospital near our home. The doctor told me that he could not treat my foot and asked me to go to another [hospital] to determine the cause. However, what kind of [hospitals] were other hospitals, and which hospital could treat my foot[?] I looked lost and did not know where to get [treatment] for my foot."

Diabetic foot complications are relatively complex, and some doctors cannot provide timely and correct diagnoses, treatments, and referrals, which could be a reason for the challenging diagnostic and treatment process. A man in his 50s and woman in her 70s reported the following, respectively:

"When we went to the hospital, the doctor treated my foot lesion as a corn. He changed the dressing for 8 months and did not know he was actually dealing with a diabetic foot complication. The doctor was treating a corn, causing a delay in treatment of 8 months."

"We first went to the hospital emergency department, and the doctor said that he could not cure my foot problem, and then, referred us to the surgery department. The surgeon looked at my foot and said that he was not sure how bad the toe was. He then said that we should get injections and infusions first to reduce the swelling. After a week of infusions, the surgeon [thought] that there was likely no or little effect of the infusions, so [the surgeon referred us] to the orthopedics department. The orthopedic doctor said that there was no major problem with the results of the examination, and then [referred us] to the neurology department of the hospital..."

Another reason for this challenging process might be that there is a large gap in the ability to treat diabetic foot complications at the different level medical institutions Most medical institutions do not have the technical means to treat diabetic foot complications. A male patient in his 70s noted the following:

"We first went to the community hospital to [treat] my foot, and after a period of time, the nurse who changed the dressing strongly recommended me to come to the Peking University Third Hospital [for treatment]. She said that we still needed to go to a big hospital to treat the foot. The community hospital could only change the dressing, and there was no other medical treatment. If we [want] medical treatment, we still needed to go to a big bospital for that."

\section{Difficulties in receiving professional treatment}

After patients finally found a professional hospital to treat their diabetic foot complications, they still encountered difficulty with receiving treatment. Patients reported difficulties in transportation, registration, and hospitalization in professional hospitals.

Traffic inconvenience mainly included difficulties regarding using wheelchairs to get a taxi, severe traffic jams on the way to the hospital, and long waiting times after entering the hospital. A man in his 50 s and woman in her 70 s commented the following, respectively:

"Every time we came to the hospital, it was not easy. Every time we came, we had to take a taxi, but it was too difficult for me to take a taxi. Because I need to take a wheelchair, the taxi driver 
did not want to take us, and [said our wheelchair was too big]. We [waited] for a long time without a taxi to [take us]. Drivers [were] willing to let those young people take the taxis but wound not let us take [one]."

"It was extremely difficult to come to the hospital. The road in front of the hospital was particularly blocked. Every time you had to come early, if you came late, you would be stuck in traffic for a long time. [When] I [approached] the door of the hospital, I could not get in after more than 50 minutes at the door. We finally got in, but we had to wait about an bour for parking. I dared not drink water every time I came, because I was afraid of [needing to go to] the toilet. I could not enter the hospital. How could I go to the toilet if I could not enter the bospital?"

When patients initially came to the hospital to see a doctor, it was generally difficult to register. Thus, many patients failed to register for medical treatments when they came at the initial hospital visit. A man in his 40s said the following:

"When my feet I first became swollen, I came to the third bospital to register, but I didn't register, so I went back. The second time I came, I still couldn't register, so I slept in the hospital and registered to see a doctor."

Most patients needed inpatient treatments, but hospitalization was difficult, especially in large hospitals, where the total number of beds and length of stay were limited. Two men in their 70s reported the following:

"We wanted to be hospitalized, but the doctor said that there are no beds, and we could not stay in the bospital. We could only get outpatient treatment."

"Hospitalization was too much trouble. The hospital stipulates that the maximum stay is half a month. After half a month, we had to leave the hospital. We could not stay for more than a few days."

\section{Doctor-patient communication}

Problems with the current doctor-patient communication protocols include insufficient communication, an inability of the patient to understand and remember the spoken information given, and the patients' believed that existing communications were not enough and more communication with the doctor was needed.

\section{Insufficient doctor-patient communication}

Communication was lacking between doctors and patients, and medical staff had a heavy workload and thereby were unable to communicate with patients fully. A man in his 50 s noted the following:

"We wanted to communicate with the doctor, but the doctor would not talk to us at all. If you asked the doctor a question, the doctor did not respond. The doctor would change your dressing and make a list. They would say to change the medicine, take the list, and then it was over. There were many people in the back to see the doctor, so there was no time to explain [the process] to you."

\section{An inability of patients to remember and understand the spoken information}

Some patients did not remember or understand what the doctor said. This was a common problem among older patients, who often asked their family members or caregivers to communicate with their doctors. A man in his 70s explained the following:

"When the doctor told me what to do, I seemed to understand it at that time. It seemed clear. However, after a period of time, I seemed to have some memory problems, and forgot what the doctor said. Because of old age, my memory was not good, so I let my daughter talk to the doctor, and I listened by ber side."

Unmet need for understanding further disease conditions Patients often needed to communicate with medical staff. Because diabetic foot complications had complicated treatment strategies, and the conditions were prone to change, patients believed that the condition explained by the doctor was not enough. There was a need for further understanding the condition. A man in his 40 s noted the following:

"I also communicated with the doctor every time I came to the hospital, but the doctor was busy; every time he gave me a few words and the visit was over. The doctor told me how to change the dressing and walk less. But how [bad] was my illness? How long would it take to recover? What else should I pay attention to? The doctor did not tell me these things. I don't have a bottom in my heart now, and I don't know when my foot will get better."

\section{Mental burden}

Patients with diabetic foot complications face a serious mental burden. Some of the study participants would have tears in their eyes, and when they talked about their sadness, they would cry and weep.

Signs of anxiety and depression appeared in some patients. Patients with diabetic foot complications generally had serious psychological burdens, and the consequences of the diabetes brought a lot of mental pressure to the patients in our study, especially those with many complications. A man in his 40 s noted the following:

"I feel a lot of pressure. I was only in my forties, and it was just when I was in the prime of my life that something went wrong 
with my foot."

Due to the complexity of the disease and the long treatment cycles, negative emotions could not be alleviated. One woman in her 60s commented the following:

"After I got this disease, I collapsed. Generally, staying in the hospital for a week was fine if you got sick. Who [knew] this disease would never get better. The disease has been going on for more than a year, but it still [won't end]. I don't look good. I'm not happy. How could I be happy, I could not wait to commit suicide, and this is really boring."

\section{Financial strain}

Most patients generally had medical insurance. Despite this, the financial burden was still considerable, and some families faced poverty due to costs associated with the illness.

\section{Limited outpatient reimbursement}

Patient medical expenses would generally exceed the upper limit of outpatient reimbursements (RMB 20,000 or \$3,089), and the expenses incurred afterward would increase patient economic burdens. A female patient in her 60s expressed the following:

"I have already exceeded the upper limit now, and it has only been 6 months. Now I have to pay for medical treatment at my own expense, so I feel great financial pressure."

\section{The lack of Medicare coverage}

Many of the drug and surgery expenses incurred by patients were not covered by Medicare, so they needed to pay at their own expense. A woman in her 60s and man in his 60 s reported the following, respectively:

"Feksung (Polymeric long-acting antibacterial film solution material) is our common medicine, but we have to pay for it at our own expense. One bottle will cost 180 RMB (\$28), and generally one bottle will be used for a week. This is not covered by the medical insurance, so I don't know why it is not reimbursed."

"The operation cost was too expensive. It costed more than $100,000 \mathrm{RMB}(\$ 15,443)$ to perform an operation. After the reimbursement, it costed 50,000 to 60,000 RMB (\$7,722\$9266). Looking at the billing list, a lot of materials were not reimbursed."

\section{Additional expenses}

In addition to medical expenses, costs included transportation, meals, and nursing workers, etc. A woman in her 70s commented the following:

"My nursing worker takes care occurred at bome because my daughter has to go to work, and there is no way to take care of me, so my daughter bires an in-bome nurse for me."

\section{Loss of employment}

Patients with diabetic foot complications lost the opportunity to work and the ability to earn money due to the disease, which brought a heavy economic burden to themselves and their families. A male patient in his $50 \mathrm{~s}$ noted the following:

"I used to be able to drive a taxi to make money, but now I am bave an amputated foot because of this disease. There is no way to drive a taxi, and there is no way to make money."

\section{Social support}

Study patients were in great need of social support. Caregivers were in high demand but limited supply, both inside and outside the patient's family.

\section{Unmet need for caregivers}

Study patients had limited ability to perform daily activities and thus needed caregivers. Some patients required multiple caregivers that were sometimes severely understaffed. There were a few patients who did not need caregivers. A man in his 40s remarked the following:

"Every time I came to the hospital, I [needed] someone to accompany me. My son recently had a boliday, and be always accompanied me [during] these times. I have two brothers, and they will accompany me, because I can't come alone due to my [difficulty with] walking, so I need at least one person to accompany me."

\section{Family caregivers}

The main caregivers were provided by the family, usually being patient partners, children, and siblings. Some families also hired nurses to take care of the patients. A male patient in his 60s reported the following:

"My main caregivers are my wife and daughter. My wife takes care of me at home, and my daughter accompanies me to the hospital."

\section{Limited support outside of family}

Apart from family support, there was little support at other social levels and almost no support from employers, coworkers, or local communities. A male patient in his 50 s noted the following:

"Except for family and siblings, there was no belp and care from other levels...apart from your family, who would care for you and take care of you?" 


\section{Discussion}

The incidence of diabetes has increased considerably. The global prevalence of patients with diabetes mellitus has risen from $4.7 \%$ in 1980 to $8.5 \%$ in 2014 among adults (2). Diabetes can lead to diabetic foot complications, which are associated with high medical costs (3). Therefore, it is important to understand the difficulties and problems faced by patients with diabetic foot complications. To our knowledge, this is the first study using qualitative methods to better understand the barriers of patients with diabetic foot complications in China. To help improve the efficacy of health services, as well as prognostic outcomes and medical expenses of patients, our study explored patient-reported problems and barriers, which were analyzed and categorized into five main dimensions: hospital visits, doctor-patient communication, mental burden, economic burden, and social support.

Our investigation was conducted during the severe acute respiratory syndrome coronavirus 2 (SARS-CoV-2) outbreak. Because the questions were open, patients had the opportunity to make a full and comprehensive description of the effect of the SARS-CoV-2 on them. Although it is undeniable that SARS-CoV-2 has affected many patients, the difficulties presented by the current study patients were still concentrated in five dimensions. This may be because there were no patients with SARS-CoV-2 under investigation. Although the hospitals in Beijing were affected by the SARS-CoV-2, they still carried out the diagnosis and treatment of diabetic foot complications. Therefore, the main difficulties stated by the patients did not include SARS-CoV-2, or at least the virus could not be regarded as an independent factor.

We found that the difficulties encountered by patients with diabetic foot complications were very common, and most patients faced more than one difficulty, such as delayed medical treatment, mental burden, and economic burden. These difficulties brought great burden to patients and their families. The reasons for these difficulties are numerous. First of all, despite the high incidence of diabetes in China (11.6\%), diabetic foot complications are not common or frequently occurring diseases. In China, not all hospitals can offer treatment (24). The hospitals that can treat diabetic foot complications are mainly large and concentrated in large cities such as Beijing, with high economic development $(25,26)$. Most hospitals in China have not established a diabetic foot complications specialty, nor can they treat diabetic foot complications, especially in the primary hospitals. Therefore, patients with diabetic foot complications, especially those with severe cases of the disease, will generally go from hospital to hospital to see a doctor, and the process of seeing a doctor can be complicated. In addition, patient barriers may be owing to the lack of social awareness of diabetic foot complications. Lack of government awareness leads to insufficient coverage of medical insurance; lack of hospital awareness leads to the inability of many primary hospitals to treat this disease; lack of medical workers' awareness leads to lack of relevant professional knowledge to treat this disease; lack of patients' own awareness leads to delay of medical treatment (27-30). Thus, focusing the attention of the whole society on diabetic foot complications might be a fundamental strategy to reduce the associated burden on patients.

Furthermore, study findings showed that the difficulties encountered by patients were concentrated in five dimensions (hospital visits, doctor-patient communication, mental burden, economic burden, and social support) that should be resolved urgently. We found that patients with diabetic foot complications experienced delayed treatment, mainly owing to the lack of knowledge about diabetes and diabetic foot complications. Several studies similarly have noted a low level of knowledge about diabetic foot complications and unsuccessful treatment-seeking behavior in patients, which is consistent with our results $(27,30,31)$. Our findings also indicated a serious deficiency in doctorpatient communication, which may be due to the heavy workload of medical staff. An in-depth interview of Chinese patients and physicians also has shown that doctors' high volume of patients leads to a serious lack of communication between doctors and patients, and there is not enough time for doctor-patient interactions in China (32).

Additionally, during our interviews, some patients would unconsciously shed tears and cry. Patients with diabetic foot complications have a serious mental burden, including anxiety and depression. One study found that compared with patients with diabetes without foot problems, those with foot problems showed more pronounced symptoms of depression (33). Anxiety and depression are widely prevalent among such patients (34). If such negative emotions cannot be addressed, patients' adherence to medical advice will become worse, and it will be more difficult to cure the disease (35).

Furthermore, patients with diabetic foot complications face a heavy financial burden. Our study results showed that medical expenses incurred during the treatment of diabetic foot complications are relatively high, and many of them are 
not covered by medical insurance reimbursement-a finding which was not noted in other studies in China. In addition to medical expenses, we also found that patients had to pay additional expenses such as transportation costs. These expenses impose a severe financial burden on patients and their families. The more serious the condition of diabetic foot complications, the more comorbidities, the greater the economic burden, and some families face poverty due to these expenses (36).

The five dimensions of barriers (hospital visits, doctorpatient communication, mental burden, economic burden, and social support) reflect the treatment dilemma of diabetic foot complications. That is, the medical discipline is underdeveloped without a comprehensive response system. This may be because diabetic foot complications are different from most diseases, and the treatment requires the involvement of multiple specialties. Diseases treated by multidisciplinary collaboration are likely to become more common in the future (37). Therefore, further research on the needs of patients with this disease can provide helpful insight regarding modern medical treatment-oriented by patient needs and interdisciplinary collaborative treatment models. We proposed seven possible solutions for each dimension found in this study.

First, strengthen science popularization and raise the awareness of the whole society to diabetic foot complications. Through the impact of example education, we can educate patients without knowledge and patients with diabetes who do not pay attention to it so as to correct their misunderstanding and encourage them to see a doctor and reduce recurrences (30). In addition, it is also necessary to popularize the science of non-professional medical staff so that they can correctly identify a disease and provide a timely referral $(38,39)$. For older patients, we can make small lectures related to diabetic foot complications, so as to reduce the threshold of obtaining popular science knowledge.

Second, improve diabetic foot complications technology, cultivate professional talents, and promote discipline maturity. At present, the development of a diabetic foot complications specialty in China is still in its initial stage. The diabetic foot complication specialty is mainly found in large hospitals of the large cities in China, and almost no primary hospitals have a diabetic foot complication specialty. However, China is working hard to develop this specialty and improve the capacity of hospitals at all levels in the specialty cooperations and medical associations to diagnose and treat diabetic foot complications $(40,41)$.
Third, establish a multidisciplinary cooperation model to improve the efficacy of diagnosis and treatment and cure rate of patients with diabetic foot complications. Multiscientific cooperation in treating diabetes has generated an international consensus (42). Being able to treat a patient with diabetic foot complications using various and multiple specialties together could greatly improve therapeutic efficiencies, while also improving a patient's medical experience $(41,43)$.

Fourth, establishing a community and family doctor system, and achieving a personalized and professional communication program is ideal (44). The demand for improved doctor-patient communications reflects the current and serious deficiency, which can be addressed using two methods, which are to increase communication channels and improve communication methods by strengthening basic medical care in the community and establishing a family doctor system to address the reason why Chinese patients primarily seek medical treatments in tertiary medical institutions (i.e., large hospitals). Establishing patient files and a follow-up system in primary medical institutions increases the doctor-patient communication link between patients and primary medical institutions. An adoption of multidimensional communication modes, including grassroots hospital guidance, and online diagnosis and treatment assistance, can greatly increase doctor-patient communications, making the treatment process more professional and efficient (45-47).

Fifth is to strengthen the psychological patient counseling, as a patient's psychological state directly affects therapeutic effects and the quality of life (48). Patient confidence in receiving successful treatments and their willingness to actively cooperate results in a better general prognosis (49). Psychological counseling generally is based on the treatment effect of physical diseases. For diabetic foot complications that must be treated by drugs and surgery, lifestyle changes and mental state adjustments occur at the same time. These changes can cause great execution pressures in patients, producing negative emotions (e.g., patients are not up to the task) (50). Therefore, psychological counseling should cooperate with the diagnosis and treatment strategies so that patients can appreciate treatment efficacies and have hope for the future.

Sixth, patient economic burdens must be reduced. In China, the cost of patient diagnoses and treatments mainly come from medical insurance (or free medical treatment) and individual family burdens. In the future, commercial insurance could become a way to reduce the economic 
burden (51).

Seventh, increase social support. The disability rate of diabetic foot complications is high, and the family burden is heavy (52). Cultivating a professional nursing staff, establishing community nursing institutions, and establishing social volunteer systems can help create a professional social support system, and make up for the staff shortages in Chinese escort and transportation services.

There are many strengths of the current study. Our study proposed five dimensions, which were discovered by information saturation and a comprehensive investigation through interviews with more than 40 patients. Additionally, it was the first study to consider the problems and obstacles of treatment processes from the perspective of patients with diabetic foot complications in China. Furthermore, it was the first study to use qualitative research methods to explore the problems and obstacles faced by patients in China.

However, our research has some limitations. The data were collected in Beijing only and not in other parts of China, which might have influenced the transferability of the study findings to other areas. However, the barriers identified in Beijing are likely to apply to other Chinese provinces. Additionally, we only interviewed patients treated in hospitals and were unable to obtain relevant information about the problems and barriers that patients face when they do not present at hospitals for treatment. Another limitation is that patients were selected by purposive sampling. Compared with the patients who were not selected, the patients included were more willing to communicate with the study team and share their difficulties, so selection bias was present. Furthermore, the surveyed population was not representative of the overall population. The patients we interviewed were mainly from the eastern part of China. Notably, the diabetic foot complication treatments were generally concentrated in large hospitals in eastern China. Primary hospitals in central and western China are currently unable to diagnose and treat diabetic foot complications, especially severe diabetic foot complications (53). Thus, patients in the central and western parts of the country might encounter greater barriers to care, which should be explored. Future studies involving patients who are in central and western China might provide additional insights into the barriers faced by patients with diabetic foot complications. In addition, some of the needs were common patient needs, whereas some were specific to patients with diabetic feet or Chinese patients, which needs further study. Current study findings could lay a foundation for further analysis of the relationship between patient needs and treatment strategies.

\section{Conclusions}

In the current study, barriers faced by patients with diabetic foot complications in Beijing in China were concentrated in five dimensions: hospital visits, doctor-patient communications, mental burdens, economic pressures, and social supports. Future studies could use these five dimensions as a foundation to solve existing problems and difficulties and improve the prognoses and outcomes of patients with diabetic foot complications.

\section{Acknowledgments}

We thank all the people who took time to participate in our interviews. We would also thank Peking University Third Hospital, Beijing Shijitan Hospital and Beijing Hospital for their support and cooperation in helping us complete this survey.

Funding: The study was supported by the "Capital Clinical Diagnosis and Treatment Technology Research and Demonstration Application" project commissioned by the Beijing Municipal Commission of Science and Technology (No. Z191100006619016).

\section{Footnote}

Reporting Checklist: The authors have completed the MDAR and COREQ reporting checklist. Available at http://dx.doi. org/10.21037/atm-20-7569

Data Sharing Statement: Available at http://dx.doi. org/10.21037/atm-20-7569

Conflicts of Interest: All authors have completed the ICMJE uniform disclosure form (available at http://dx.doi. org/10.21037/atm-20-7569). The authors have no conflicts of interest to declare.

Etbical Statement: The authors are accountable for all aspects of the work in ensuring that questions related to the accuracy or integrity of any part of the work are appropriately investigated and resolved. The study was conducted in accordance with the Declaration of Helsinki (as revised in 2013). The ethic approval was obtained from Peking University Third Hospital Institutional Review board (No. IRB00006761-M2020003). Informed consent was obtained from the participants.

Open Access Statement: This is an Open Access article 


\section{Page 12 of 14}

distributed in accordance with the Creative Commons Attribution-NonCommercial-NoDerivs 4.0 International License (CC BY-NC-ND 4.0), which permits the noncommercial replication and distribution of the article with the strict proviso that no changes or edits are made and the original work is properly cited (including links to both the formal publication through the relevant DOI and the license). See: https://creativecommons.org/licenses/by-nc-nd/4.0/.

\section{References}

1. Sen P, Demirdal T. Evaluation of mortality risk factors in diabetic foot infections. Int Wound J 2020;17:880-9.

2. Zhang $Z$, Cui $T$, Cui $M$, et al. High prevalence of chronic kidney disease among patients with diabetic foot: A crosssectional study at a tertiary hospital in China. Nephrology (Carlton) 2020;25:150-5.

3. Zhou Q, Peng M, Zhou L, et al. Development and validation of a brief diabetic foot ulceration risk checklist among diabetic patients: a multicenter longitudinal study in China. Sci Rep 2018;8:962.

4. Ma RC. Epidemiology of diabetes and diabetic complications in China. Diabetologia 2018;61:1249-60.

5. Saeedi P, Petersohn I, Salpea P, et al. Global and regional diabetes prevalence estimates for 2019 and projections for 2030 and 2045: Results from the International Diabetes Federation Diabetes Atlas, 9(th) edition. Diabetes Res Clin Pract 2019;157:107843.

6. Jiang $\mathrm{Y}$, Wang $\mathrm{X}$, Xia L, et al. A cohort study of diabetic patients and diabetic foot ulceration patients in China. Wound Repair Regen 2015;23:222-30.

7. Adeloye D, Ige JO, Aderemi AV, et al. Estimating the prevalence, hospitalisation and mortality from type 2 diabetes mellitus in Nigeria: a systematic review and metaanalysis. BMJ Open 2017;7:e015424.

8. Al-Rubeaan K, Almashouq MK, Youssef AM, et al. Allcause mortality among diabetic foot patients and related risk factors in Saudi Arabia. PLoS One 2017;12:e0188097.

9. Foster D, Lauver LS. When a diabetic foot ulcer results in amputation: a qualitative study of the lived experience of 15 patients. Ostomy Wound Manage 2014;60:16-22.

10. Hingorani A, LaMuraglia GM, Henke $P$, et al. The management of diabetic foot: A clinical practice guideline by the Society for Vascular Surgery in collaboration with the American Podiatric Medical Association and the Society for Vascular Medicine. J Vasc Surg 2016;63:3S-21S.

11. Chapman A, Yang H, Thomas SA, et al. Barriers and

\section{Liu et al. Barriers of patients with diabetic foot complications}

enablers to the delivery of psychological care in the management of patients with type 2 diabetes mellitus in China: a qualitative study using the theoretical domains framework. BMC Health Serv Res 2016;16:106.

12. Barg FK, Cronholm PF, Easley EE, et al. A qualitative study of the experience of lower extremity wounds and amputations among people with diabetes in Philadelphia. Wound Repair Regen 2017;25:864-70.

13. Palaya J, Pearson S, Nash T. Perception of social support in individuals living with a diabetic foot: A qualitative study. Diabetes Res Clin Pract 2018;146:267-77.

14. Coffey L, Mahon C, Gallagher P. Perceptions and experiences of diabetic foot ulceration and foot care in people with diabetes: A qualitative meta-synthesis. Int Wound J 2019;16:183-210.

15. The L. A tiered health-care delivery system for China. Lancet 2019;393:1178.

16. Chen J, Xu S, Gao J. The Mixed Effect of China's New Health Care Reform on Health Insurance Coverage and the Efficiency of Health Service Utilisation: A Longitudinal Approach. Int J Environ Res Public Health 2020;17:1782.

17. Zhou S, Xu J, Ma X, et al. How Can One Strengthen a Tiered Healthcare System through Health System Reform? Lessons Learnt from Beijing, China. Int J Environ Res Public Health 2020;17:8040.

18. Tao F, Tang X, Tao H, et al. Surgical treatment of diabetic foot ulcers during the COVID-19 pandemic in China. J Diabetes Complications 2020;34:107622.

19. Guell C, Unwin N. Barriers to diabetic foot care in a developing country with a high incidence of diabetes related amputations: an exploratory qualitative interview study. BMC Health Serv Res 2015;15:377.

20. Mogre V, Johnson NA, Tzelepis F, et al. Barriers to diabetic self-care: A qualitative study of patients' and healthcare providers' perspectives. J Clin Nurs 2019;28:2296-308.

21. Fayfman M, Schechter MC, Amobi CN, et al. Barriers to diabetic foot care in a disadvantaged population: A qualitative assessment. J Diabetes Complications 2020;34:107688.

22. Zhang P, Lu J, Jing Y, et al. Global epidemiology of diabetic foot ulceration: a systematic review and metaanalysis (dagger). Ann Med 2017;49:106-16.

23. Tong A, Sainsbury P, Craig J. Consolidated criteria for reporting qualitative research (COREQ): a 32-item checklist for interviews and focus groups. Int J Qual Health Care 2007;19:349-57. 
24. Jiang Y, Ran X, Jia L, et al. Epidemiology of type 2 diabetic foot problems and predictive factors for amputation in China. Int J Low Extrem Wounds 2015;14:19-27.

25. Wang A, Xu Z, Mu Y, et al. Clinical characteristics and medical costs in patients with diabetic amputation and nondiabetic patients with nonacute amputation in central urban hospitals in China. Int J Low Extrem Wounds 2014;13:17-21.

26. Li Q, Wang X, Hu X, et al. A survey of diabetic foot patients with hospitalized amputations performed in a medical center over 5 years in China: limitations and lessons learnt. Int J Low Extrem Wounds 2012;11:194-200.

27. Nickinson AT, Bridgwood B, Houghton J, et al. A systematic review investigating the identification, causes, and outcomes of delays in the management of chronic limb-threatening ischemia and diabetic foot ulceration. J Vasc Surg 2020;71:669-81.e2.

28. Xu Z, Ran X. Diabetic foot care in China: challenges and strategy. Lancet Diabetes Endocrinol 2016;4:297-8.

29. Daemmrich A. The political economy of healthcare reform in China: negotiating public and private. Springerplus 2013;2:448.

30. Abbas ZG, Archibald LK. Tropical diabetic hand syndrome. Epidemiology, pathogenesis, and management. Am J Clin Dermatol 2005;6:21-8.

31. Mentock SM, Ng VY, Narayana R, et al. Treatmentseeking behavior and obstacles to treatment compliance in diabetic patients in Mangaluru, India. Diabetes Metab Syndr 2017;11 Suppl 2:S617-22.

32. Ting X, Yong B, Yin L, et al. Patient perception and the barriers to practicing patient-centered communication: A survey and in-depth interview of Chinese patients and physicians. Patient Educ Couns 2016;99:364-9.

33. Hoban C, Sareen J, Henriksen CA, et al. Mental health issues associated with foot complications of diabetes mellitus. Foot Ankle Surg 2015;21:49-55.

34. Ahmad A, Abujbara M, Jaddou H, et al. Anxiety and Depression Among Adult Patients With Diabetic Foot: Prevalence and Associated Factors. J Clin Med Res 2018;10:411-8.

35. Mensah GA, Collins PY. Understanding mental health for the prevention and control of cardiovascular diseases. Glob Heart 2015;10:221-4.

36. Wu H, Eggleston KN, Zhong J, et al. How do type 2 diabetes mellitus (T2DM)-related complications and socioeconomic factors impact direct medical costs? A cross-sectional study in rural Southeast China. BMJ Open 2018;8:e020647.

37. Brown S, Nixon J, Ransom M, et al. Multiple Interventions for Diabetic Foot Ulcer Treatment Trial (MIDFUT): study protocol for a randomised controlled trial. BMJ Open 2020;10:e035947.

38. Kelly JF, Fallah-Sohy N, Vilsaint C, et al. New kid on the block: An investigation of the physical, operational, personnel, and service characteristics of recovery community centers in the United States. J Subst Abuse Treat 2020;111:1-10.

39. Kaminsky J, Gadaleta D. A study of discrimination within the medical community as viewed by obese patients. Obes Surg 2002;12:14-8.

40. Cheng Y, Zu P, Zhao J, et al. Differences in initial versus recurrent diabetic foot ulcers at a specialized tertiary diabetic foot care center in China. J Int Med Res 2021;49:300060520987398.

41. Wang A, Lv G, Cheng X, et al. Guidelines on multidisciplinary approaches for the prevention and management of diabetic foot disease (2020 edition). Burns Trauma 2020;8:a017.

42. Liu Y, Shi Y, Zhu J,et al. Study on the Effect of the Fivein-One Comprehensive Limb Salvage Technologies of Treating Severe Diabetic Foot. Adv Wound Care (New Rochelle) 2020;9:676-85.

43. Wang G, Wang L, Wang Y,et al. Multidisciplinary approach to scheduling surgery for diabetic foot: a case report. BMC Musculoskelet Disord 2019;20:168.

44. Asghari S, Kirkland MC, Blackmore J,et al. A systematic review of reviews: Recruitment and retention of rural family physicians. Can J Rural Med 2020;25:20-30.

45. Uçkay I, Gariani K, Dubois-Ferriere V, et al. Diabetic foot infections: recent literature and cornerstones of management. Curr Opin Infect Dis 2016;29:145-52.

46. An LC, Schillo BA, Saul JE, et al. Utilization of smoking cessation informational, interactive, and online community resources as predictors of abstinence: cohort study. J Med Internet Res 2008;10:e55.

47. Dadds MR, Thai C, Mendoza DA, et al. Therapistassisted online treatment for child conduct problems in rural and urban families: Two randomized controlled trials. J Consult Clin Psychol 2019;87:706-19.

48. Ross S, Bossis A, Guss J,et al. Rapid and sustained symptom reduction following psilocybin treatment for anxiety and depression in patients with life-threatening cancer: a randomized controlled trial. J Psychopharmacol 2016;30:1165-80.

49. Sehati Shafaee F, Mirghafourvand M, Harischi S,et al. Self- 


\section{Page 14 of 14}

Confidence and Quality of Life in Women Undergoing Treatment for Breast Cancer. Asian Pac J Cancer Prev 2018;19:733-40.

50. Hoban C, Sareen J, Henriksen CA,et al. Mental health issues associated with foot complications of diabetes mellitus. Foot Ankle Surg 2015;21:49-55.

51. Liu J, Chen H, Chen Y, et al. Exploring the relationship between migrants' purchasing of commercial medical

Cite this article as: Liu X, Chu H, Zhao J, Qiao R, Liu Y, Li N, Zeng L, Wang X, Tao L, Zhang H, Shi Y, Zhuo L, Zhang L, Zhao Y. Exploring the barriers of patients with diabetic foot complications in China: a qualitative interview study. Ann Transl Med 2021;9(9):792. doi: 10.21037/atm-20-7569

\section{Liu et al. Barriers of patients with diabetic foot complications}

insurance and urbanisation in China. BMC Health Serv Res 2018;18:679.

52. Wei ZR, Huang GT. Progress in the treatment of chronic wound and discussion on the integrated surgical wound treatment mode. Zhonghua Shao Shang Za Zhi 2019;35:824-7.

53. Li X, Lu J, Hu S, et al. The primary health-care system in China. Lancet 2017;390:2584-94. 


\section{Supplementary}

\section{Supplementary file 1 Topic guide}

1. Please tell me about your experience of seeing a doctor for diabetic foot.

- How did your wound come about?

- When did you first notice a problem with your foot?

- Did you go to see a doctor in time after it happened?

- What was wrong with your foot that you should see a doctor about? How long did it take you from the time you noticed the foot problem to the time you went to the doctor?

- Which hospital did you go to first? Which department did you go to? What treatment did the doctor take?

- How about the treatment effect? Which hospital did you go to after that? Which department did you go to? How about the treatment effect?.......

2. From the time you found out that there was a problem with your feet until you came to the hospital, do you think you should see a doctor in time? If you think it was not in time, what do you think was the reason that restricted your timely medical treatment?

3. When you first came to the hospital, did you have a good treatment? Was it inconvenient for you? What inconvenience did it bring to you?

4. Please tell me about your communication process about diabetic foot during your medical treatment.

- During your treatment of diabetic foot, do you often communicate with medical staff? What questions do you ask? What do you want to learn from your doctor?

- Did you communicate well with the medical staff? What problems and difficulties did you encounter in the process of communication?

5. What are the main medical expenses incurred during your treatment of diabetic foot? Can these expenses be reimbursed through medical insurance? What percentage can be reimbursed? In addition to medical expenses, what other expenses did you have during the treatment of diabetic foot?

6. Do you need someone to take care of you after your diabetic foot? In what ways do you need someone to take care of you? How many people do you need to come with you every time you come to the hospital?

7. How do you feel after you get diabetic foot? How do you feel about it?

8. In the process of treating diabetic foot, what are your main concerns? What inconvenience does diabetic foot bring to you? What inconvenience does the current treatment bring to you? 
Supplementary file 2 Themes, subthemes and example quotes

\begin{tabular}{|c|c|c|c|}
\hline Themes & Subthemes & Causes & Example quotes \\
\hline \multirow[t]{3}{*}{ Hospital visits } & $\begin{array}{l}\text { Delayed } \\
\text { treatments }\end{array}$ & $\begin{array}{l}\text { The initial disease was } \\
\text { insidious and easy to } \\
\text { ignore }\end{array}$ & $\begin{array}{l}\text { "At the beginning, I didn't notice the swollen leg or a wound on my foot, because I could walk. Because of diabetes, my peripheral nerves are bac } \\
\text { and I cannot see clearly. I cannot see or feel pain. When my daughter came to see me, she found that my leg was swollen and there was a small } \\
\text { hole in the sole of my right foot... } 28 \text { days had already passed when my daughter found these problems; and then, she hurriedly took me to the } \\
\text { hospital."(Female patient in her } 60 \mathrm{~s} \text { ) }\end{array}$ \\
\hline & & $\begin{array}{l}\text { Lack of knowledge } \\
\text { about diabetic foot }\end{array}$ & $\begin{array}{l}\text { "When my right foot first started to turn black, I thought it was bruised and okay. I felt it would just get better little by little. After more than a mont } \\
\text { my right foot did not get better, and I thought something might be wrong, so I went to the hospital to have it examined." (Male patient in his } 40 \text { s) }\end{array}$ \\
\hline & & $\begin{array}{l}\text { Lack of timely } \\
\text { knowledge acquisition }\end{array}$ & $\begin{array}{l}\text { "I did not know about diabetic foot complications before. After I came here, the doctor told me that I [had] diabetic foot complications. I didn't kn } \\
\text { it before." (Male patient in his 60s, LZD) } \\
\text { "I did not know what this disease was before, and now I do not know what it is. I just need to know how to eat, and other things are not clear. My } \\
\text { daughter knows these things. I just want to get better soon." (Female patient in her 70s, TCY) }\end{array}$ \\
\hline
\end{tabular}

A challenging Poor access to medical "My foot was so painful that I went to the community hospital near our home. The doctor told me that he could not treat my foot and asked me to go process of information to another [hospital] to determine the cause. However, what kind of [hospitals] were other hospitals, and which hospital could treat my foot[?] I looked diagnostics and initial treatments

Lack of capacity for "When we went to the hospital, the doctor treated my foot lesion as a corn. He changed the dressing for 8 months and did not know he was actually identification, diagnosis, dealing with a diabetic foot complication. The doctor was treating a corn, causing a delay in treatment of 8 months." (Male patient in his 50s, SHB) treatment and referral in "We first went to the hospital emergency department, and the doctor said that he could not cure my foot problem, and then, referred us to the some doctors surgery department. The surgeon looked at my foot and said that he was not sure how bad the toe was. He then said that we should get injections and infusions first to reduce the swelling. After a week of infusions, the surgeon [thought] that there was likely no or little effect of the infusions, so [the surgeon referred us] to the orthopedics department. The orthopedic doctor said that there was no major problem with the results of the examination, and then [referred us] to the neurology department of the hospital..." (Female patient in her 70s, ZWY)

A big gap between "We first went to the community hospital to [treat] my foot, and after a period of time, the nurse who changed the dressing strongly recommended different levels of me to come to the Peking University Third Hospital [for treatment]. She said that we still needed to go to a big hospital to treat the foot. The medical institutions community hospital could only change the dressing, and there was no other medical treatment. If we [want] medical treatment, we still needed to go to a big hospital for that." (Male patient in his 70s, GQS)

Difficulties
in receiving

Poor transportation "Every time we came to the hospital, it was not easy. Every time we came, we had to take a taxi, but it was too difficult for me to take a taxi. Because

professional

treatment

I need to take a wheelchair, the taxi driver did not want to take us, and [said our wheelchair was too big]. We [waited] for a long time without a taxi to [tak us]. Drivers [were] willing to let those young people take the taxis but wound not let us take [one]." (Male patient in his 50s, SHB)

"It was extremely difficult to come to the hospital. The road in front of the hospital was particularly blocked. Every time you had to come early, if you came late, you would be stuck in traffic for a long time. [When] I [approached] the door of the hospital, I could not get in after more than 50 minutes at the door. We finally got in, but we had to wait about an hour for parking. I dared not drink water every time I came, because I was afraid of [needing to go to] the toilet. I could not enter the hospital. How could I go to the toilet if I could not enter the hospital?" (Female patient in her 70s, HFQ)

Difficult to register "When my feet I first became swollen, I came to the third hospital to register, but I didn't register, so I went back. The second time I came, I still couldn't register, so I slept in the hospital and registered to see a doctor." (Male patient in his 40s, LY)

Difficulties in "We wanted to be hospitalized, but the doctor said that there are no beds, and we could not stay in the hospital. We could only get outpatient hospitalization treatment." (Male patient in his 70s, LXY)

"Hospitalization was too much trouble. The hospital stipulates that the maximum stay is half a month. After half a month, we had to leave the hospital. We could not stay for more than a few days." (Male patient in his 70s, HYT)

Doctor-patient Insufficient

"We wanted to communicate with the doctor, but the doctor would not talk to us at all. If you asked the doctor a question, the doctor did not respond. The doctor would change your dressing and make a list. They would say to change the medicine, take the list, and then it was over. There were many people in the back to see the doctor, so there was no time to explain [the process] to you." (Male patient in his 50s, HCS)

An inability

of patients to

remember and

understand

the spoken

information

Unmet need for understanding further disease conditions

mental burden Mental burden existed, and anxiety and depression appeared in some patients Long-term negative emotions that cannot be alleviated

Limited outpatient reimbursement

The lack of Medicare coverage

"When the doctor told me what to do, I seemed to understand it at that time. It seemed clear. However, after a period of time, I seemed to have some memory problems, and forgot what the doctor said. Because of old age, my memory was not good, so I let my daughter talk to the doctor, and I listened by her side." (Male patient in his 70s, WHW)

"I also communicated with the doctor every time I came to the hospital, but the doctor was busy; every time he gave me a few words and the visit was over. The doctor told me how to change the dressing and walk less. But how [bad] was my illness? How long would it take to recover? What else should I pay attention to? The doctor did not tell me these things. I don't have a bottom in my heart now, and I don't know when my foot will get better." (Male patient in his 40s, LSF)

"I feel a lot of pressure. I was only in my forties, and it was just when I was in the prime of my life that something went wrong with my foot."(Male patient in his 40 s, XLJ)

"After I got this disease, I collapsed. Generally, staying in the hospital for a week was fine if you got sick. Who [knew] this disease would never get better. The disease has been going on for more than a year, but it still [won't end]. I don't look good. I'm not happy. How could I be happy, I could not wait to commit suicide, and this is really boring." (Female patient in her 60s, WCS)

"I have already exceeded the upper limit now, and it has only been 6 months. Now I have to pay for medical treatment at my own expense, so I feel great financial pressure." (Female patient in her 60s, LCP)

"Jeksung (Polymeric long-acting antibacterial film solution material) is our common medicine, but we have to pay for it at our own expense. One bottle will cost 180 RMB (\$28), and generally one bottle will be used for a week. This is not covered by the medical insurance, so I don't know why it is not reimbursed." (Female patient in her 60s, ZHR)

"The operation cost was too expensive. It costed more than 100,000 RMB $(\$ 15,443)$ to perform an operation. After the reimbursement, it costed 50,000 to 60,000 RMB (\$7,722-\$9266). Looking at the billing list, a lot of materials were not reimbursed." (Male patient in his 60s, SBZ)

Additional expenses for

transportation,

meals, nursing workers, etc.

Loss of

employment

Social support Unmet needs of caregivers, and few patients who do not need caregivers

Almost all

caregivers

currently provided by the family "My nursing worker takes care occurred at home because my daughter has to go to work, and there is no way to take care of me, so my daughter hires an in-home nurse for me." (Female patient in her 70s, HFQ)

"I used to be able to drive a taxi to make money, but now I am have an amputated foot because of this disease. There is no way to drive a taxi, and there is no way to make money." (Male patient in his 50s, SHB)

"Every time I came to the hospital, I [needed] someone to accompany me. My son recently had a holiday, and he always accompanied me [during] these times. I have two brothers, and they will accompany me, because I can't come alone due to my [difficulty with] walking, so I need at least one person to accompany me."(Male patient in his 40s, QZS)

"My main caregivers are my wife and daughter. My wife takes care of me at home, and my daughter accompanies me to the hospital." (Male patient in his 70s, SSZ) 